ANNALES

POLONICI MATHEMATICI

$82.2(2003)$

\title{
On locally biholomorphic surjective mappings
}

\author{
by EwA LigockA (Warszawa)
}

\begin{abstract}
We prove that each open Riemann surface can be locally biholomorphically (locally univalently) mapped onto the whole complex plane. We also study finite-toone locally biholomorphic mappings onto the unit disc. Finally, we investigate surjective biholomorphic mappings from Cartesian products of domains.
\end{abstract}

0. Introduction. The motivation for writing this note came from the book [6]. We find there the Gunning-Narasimhan theorem which says that each open Riemann surface can be expressed as a Riemann domain over $\mathbb{C}$. Moreover, the whole book [6] is devoted to the study of holomorphic functions on Riemann domains over $\mathbb{C}^{n}$.

If $X$ is a complex connected manifold and $f$ is a locally biholomorphic map from $X$ onto $\mathbb{C}^{n}$, then the pair $(X, f)$ forms a Riemann domain over $\mathbb{C}^{n}$.

The natural question arises: What can we say about the image of $X$ under a locally biholomorphic map $f: X \rightarrow \mathbb{C}^{n}$ ? In particular, for which $X$ can we find such an $f$ with $f(X)=\mathbb{C}^{n}$ ?

The main result of the present note is that for every open Riemann surface there exists a locally biholomorphic mapping from $X$ onto $\mathbb{C}$. This implies that every Cartesian product of $n$ such surfaces can be thought of as a Riemann domain over the whole $\mathbb{C}^{n}$. We do not know if this is true for every Riemann-Stein domain over $\mathbb{C}^{n}$.

In the rest of this paper we study locally biholomorphic mappings with finite fibers. We give geometric conditions on a domain $D \subset \mathbb{C}$ sufficient for the existence of a locally biholomorphic mapping with finite fibers from $D$ onto the unit disc. The Fornæss-Stout theorem [1] implies that such a domain can be mapped locally biholomorphically onto each Riemann surface by a mapping with finite fibers.

2000 Mathematics Subject Classification: Primary 30C55, 30F99, 32H02.

Key words and phrases: Riemann surface, Gunning-Narasimhan theorem, Riemann domain, locally biholomorphic, Riemann-Stein domain. 
We shall use standard notations. The symbol $\langle a, b\rangle$ stands for the closed interval with ends $a$ and $b$. We deal with domains in $\mathbb{C}^{n}$ or $n$-dimensional complex manifolds.

1. The case of $n=1$. We start with the following

TheOREM 1. For every entire function $g \neq$ const and every domain $D \subset \mathbb{C}$ there exists a homography $h$ such that the function

$$
F(z)=(\exp (g \circ h)-1) \exp (\exp (g \circ h))
$$

maps $D$ onto $\mathbb{C}$. If $g^{\prime}(z) \neq 0$ on $\mathbb{C}$ then $F^{\prime}(z) \neq 0$ on $D$ and thus $F$ is a local biholomorphism.

Proof. The function $\varphi(z)=(z-1) e^{z}$ maps $\mathbb{C} \backslash\{0\}$ onto $\mathbb{C}$ by the Picard theorem. The derivative $\varphi^{\prime}(z)=z e^{z}$ vanishes only at zero. The function $e^{g}$ has an essential singularity at $\infty$. Hence $e^{g(1 / z)}$ has an essential singularity at zero. The Julia theorem ([7], see also [3, Ch. $2, \S 7$, Th. 5]) implies that there exists a halfline $l$ with end at zero such that for every open neighborhood $U$ of zero and every open angle $A$ with bisectrix $l$ the function $e^{g(1 / x)}$ takes every value from $\mathbb{C} \backslash\{0\}$ on $U \cap A$. Let $l^{\perp}$ denote the line through zero perpendicular to $l$, and let $H$ be the half-plane with $\partial H=l^{\perp}$ and $l \subset H$.

Now let $D$ be a domain in $\mathbb{C}$. If $D=\mathbb{C}$ then it suffices to put $h(z)=z$. The function

$$
F=\varphi \circ e^{g}=(\exp g-1) \exp (\exp g)
$$

is as required.

If $D \neq \mathbb{C}$ then we can take $a \in D$ and $b \in \partial D$ such that

$$
\varrho=\operatorname{dist}(a, \partial D)=|a-b| \text {. }
$$

Let $\Delta$ denote the open disc with center $a$ and radius $\varrho$. We can find a homography $h_{1}$ which maps $\Delta$ onto $H$ such that $h_{1}(b)=0$ (note that each conformal map from a disc onto a half-plane must be a homography). Let $h=1 / h_{1}$. Then $e^{g \circ h}$ maps $D$ onto $\mathbb{C} \backslash\{0\}$ and hence

$$
F=\varphi \circ e^{g \circ h}=(\exp (g \circ h)-1) \exp (\exp (g \circ h))
$$

maps $D$ onto $\mathbb{C}$. We have

$$
F^{\prime}(z)=\varphi^{\prime}(\exp (g \circ h)(z)) \cdot \exp (g \circ h)(z) \cdot g^{\prime}(h(z)) \cdot h^{\prime}(z)
$$

and $F^{\prime}(z)=0$ if $g^{\prime}(h(z))=0$.

Corollary 1. For each domain $D \subset \mathbb{C}$ there exists a homography $h$ such that

$$
F_{h}(z)=(\exp h(z)-1) \exp (\exp h(z))
$$

maps $D$ onto $\mathbb{C}$ and $F^{\prime}(z) \neq 0$ for $z \in D$.

Proof. Put $g(z)=z$ in Theorem 1. 
Corollary 2. Let $X$ be a connected complex manifold and let $f: X \rightarrow \mathbb{C}$ be a nonconstant holomorphic function. There exists a homography $h$ such that the mapping $F_{h} \circ f$ maps $X$ onto $\mathbb{C}$.

Proof. Apply Corollary 1 to the domain $D=f(X)$.

The term "open Riemann surface" will stand for "one-dimensional connected, noncompact complex manifold".

Our Corollary 2 yields in particular the following

THEOREM 2. For each open Riemann surface $X$ there exists a locally biholomorphic map $p$ from $X$ onto $\mathbb{C}$.

In other words, $X$ can be represented as a Riemann domain over the whole plane $\mathbb{C}$.

Proof. By the Gunning-Narasimhan theorem ([4], see also [6, Ch. 1, $\S 1.11])$ there exists a locally biholomorphic mapping $\varphi$ from $X$ into $\mathbb{C}$. We can now apply Corollary 2.

The mappings constructed in Theorems 1 and 2 were all infinite-toone. Thus a natural question arises: Does there exist a finite-to-one locally biholomorphic mapping from $X$ onto $\mathbb{C}$ ?

We have the following

Proposition 1. Let $D=\mathbb{C} \backslash\{0\}$ and let $f: \mathbb{C} \backslash\{0\} \rightarrow \mathbb{C}$ be a locally biholomorphic map onto $\mathbb{C}$. Then for all $w \in \mathbb{C}$ except at most one, the set $f^{-1}(w)$ is infinite.

To prove this we shall need the following

LEMMA 1. If $R$ is a rational function which maps $\mathbb{C} \backslash\{0\}$ onto $\mathbb{C}$, then there exists $z_{0} \in \mathbb{C} \backslash\{0\}$ such that $R^{\prime}\left(z_{0}\right)=0$.

Proof of Lemma 1. The derivative $R^{\prime}(z)$ is rational and holomorphic on $\mathbb{C} \backslash\{0\}$. The Laurent series of $R^{\prime}(z)$ at zero has the form

$$
R^{\prime}(z)=\sum_{k=0}^{N} a_{k} z^{k}+\sum_{k=1}^{M} a_{-k} z^{-k}
$$

If $R^{\prime}(z) \neq 0$ on $\mathbb{C} \backslash\{0\}$ then $R^{\prime}$ must be zero either at 0 or at $\infty$. In the first case

$$
R^{\prime}(z)=a_{N} z^{N}, \quad R(z)=\frac{a_{N} z^{N+1}}{N+1}+c
$$

and therefore $R(z) \neq c$ for $z \in \mathbb{C} \backslash\{0\}$. In the second case

$$
R^{\prime}(z)=a_{-M} z^{-M}, \quad M \neq 1, \quad R(z)=\frac{a_{-M} z^{-M+1}}{-M+1}+c
$$

and again $R(z) \neq c$ on $\mathbb{C} \backslash\{0\}$. In both cases we have obtained a contradiction. 
Proof of Proposition 1. If there exist two different values $w_{1}$ and $w_{2}$ such that the sets $\left\{f^{-1}\left(w_{1}\right)\right\}$ and $\left\{f^{-1}\left(w_{2}\right)\right\}$ are finite then $f$ has an essential singularity neither at 0 nor at $\infty$ (Picard theorem). Thus $f$ must be rational, which contradicts Lemma 1.

Definition 1. Let $f: X \stackrel{\text { onto }}{\longrightarrow} Y$. We shall say that $f$ is $m$-valent, $m \in \mathbb{N}$, if for each $y \in Y$ the set $\left\{f^{-1}(g)\right\}$ has no more than $m$ elements.

It turns out that the case of $D=\mathbb{C} \backslash\{0\}$ is in some sense exceptional:

Theorem 3. Let $D$ be a finitely connected domain in $\mathbb{C}$, not biholomorphic to $\mathbb{C} \backslash\{0\}$. Then there exist $m \in \mathbb{N}$ and an m-valent locally biholomorphic mapping $f$ from $D$ onto $\mathbb{C}$.

Proof. We shall consider several cases:

(a) $D=\mathbb{C} \backslash\left\{a_{1}, \ldots, a_{N}\right\}, a_{i} \neq a_{j}, i \neq j, N \geq 2$. Let $M \in \mathbb{N}, M \geq N$, be chosen such that

$$
\frac{M+1}{M+2} \cdot \frac{a_{i}-a_{1}}{a_{2}-a_{1}} \neq 1 \quad \text { for } 2 \leq i \leq N .
$$

Let $\varphi(z)=z^{M+1}(z-1)$ and let $h$ be a linear mapping sending $a_{1}$ to 0 and $a_{2}$ to $\frac{M+1}{M+2}$. Put $f=\varphi \circ h$. We have $f^{\prime}(z) \neq 0$ on $D$. Moreover $1 \in h(D)$ and $f\left(h^{-1}(1)\right)=0$. Any other value $w \neq 0$ in $\mathbb{C}$ is taken by $\varphi$ at $\geq M+1$ different points. Hence $f$ maps $D$ onto $\mathbb{C}$. The mapping $f$ is $(M+2)$-valent.

(b) $D=B(0,1)$, the unit disc. We can construct $f$ as the superposition $f=f_{4} \circ f_{3} \circ f_{2} \circ f_{1}$, where $f_{1}=h^{3}, h$ is a biholomorphic map of $B(0,1)$ onto the upper halfdisc and

$$
f_{2}(z)=\frac{1}{2}\left(z+\frac{1}{z}\right), \quad f_{3}(z)=\frac{z}{\sqrt{3}}, \quad f_{4}(z)=z^{3}-z .
$$

Note that $f_{1}$ maps $B(0,1)$ onto $B(0,1) \backslash\{0\}, f_{3} \circ f_{2} \circ f_{1}$ maps $B(0,1)$ onto $\mathbb{C} \backslash\langle-1 / \sqrt{3}, 1 / \sqrt{3}\rangle$ and $f_{4}(z)=z^{3}-z$ maps $\mathbb{C} \backslash\langle-1 / \sqrt{3}, 1 / \sqrt{3}\rangle$ onto $\mathbb{C}$. It is easy to check that $f^{\prime}(z) \neq 0$ on $D$. Thus by the Riemann theorem we have already proved the assertion of Theorem 3 for all simply connected domains.

(c) If $D$ is a $k$-connected domain, $k \geq 2$, and $D$ is not biholomorphic to any of the domains from (a) then by the Koebe theorem (see [3, Ch. $5, \S 6$, Th. 2 and remarks at the end of $\S 6]), D$ is biholomorphic to the annulus $A=\{z: 0 \leq r<|z|<1\}$ with $k-2$ closed disc or points removed. There exists an open angle $\alpha,|\alpha|=2 \pi k / q, k / q \in \mathbb{Q}$, such that $\alpha \cap A=$ $\alpha \cap\{$ biholomorphic image of $D\}$. Hence $g(z)=z^{q+1}$ maps the biholomorphic image of $D$ onto the whole annulus $A$. Thus we have obtained a locally biholomorphic $(q+1)$-valent mapping $D$ onto $A$.

It now suffices to find $m \in \mathbb{N}$ and an $m$-valent locally biholomorphic mapping $A$ onto $\mathbb{C}$. If $r=0$ then $A=B(0,1) \backslash\{0\}$ and we can take the 
superposition $f_{4} \circ f_{3} \circ f_{2}$, where $f_{i}, i=2,3,4$, are the mappings from item (b). If $r>0$ we can take the mapping $\varphi_{r}(z)=\frac{z-r}{1-r z}$, use the function $\varphi_{r}^{3}$ which maps $A$ onto $B(0,1) \backslash\{0\}$, and proceed as before.

If our domain $D$ is biholomorphic to a bounded domain we can ask whether there exists an $m$-valent locally biholomorphic mapping $f$ from $D$ onto the unit disc $B(0,1)$.

We start with the following

Proposition 2. Let $D$ be a domain contained in $B(0,1)$. Assume that there exist $z_{0} \in \partial B(0,1)$ and $r>0$ such that

$$
D \cap B\left(z_{0}, r\right)=B(0,1) \cap B\left(z_{0}, r\right) .
$$

Then there exists a locally biholomorphic map $f$ from $D$ onto $B(0,1)$ which is $m$-valent with $m \leq 24$.

Proof. By the Riemann theorem we can find a biholomorphic mapping $h$ from $B(0,1)$ onto the strip

$$
\left\{z: \operatorname{Re} z<0,|\operatorname{Im} z|<\frac{3}{2} \pi\right\}
$$

which extends to $\overline{B(0,1)}$ in such a way that the arc

$$
B\left(z_{0}, r\right) \cap \partial B(0,1)
$$

is mapped onto

$$
\left\langle-a-\frac{3}{2} \pi i,-\frac{3}{2} \pi i\right\rangle \cup\left\langle-\frac{3}{2} \pi i, \frac{3}{2} \pi i\right\rangle \cup\left\langle\frac{3}{2} \pi i, \frac{3}{2} \pi i-a\right\rangle, \quad a>0 .
$$

Thus the set $h\left(B(0,1) \cap B\left(z_{0}, r\right)\right)$ contains a rectangle $R$ with vertices $\left(-b-\frac{3}{2} \pi i,-\frac{3}{2} \pi i, \frac{3}{2} \pi i, \frac{3}{2} \pi i-b\right)$ for some $b$ with $0<b<a$. By assumption we have $R \subset h(D)$. Hence $g=e^{h}$ is a 2-valent locally biholomorphic mapping from $D$ into $B(0,1)$ such that $B(0,1) \backslash g(D) \subset B\left(0, e^{-b}\right)$. Let $r=\max (|z|: z \in B(0,1) \backslash g(D))$ and let $z_{0} \in B(0,1) \backslash g(D)$ be such that $\left|z_{0}\right|=r$. Put

$$
\varphi=\frac{z-z_{0}}{1-\bar{z}_{0} z} .
$$

Then $\varphi^{3}$ is a 3 -valent locally biholomorphic mapping from $g(D)$ onto $B(0,1) \backslash\{0\}$ (cf. (c) of the proof of Theorem 3).

Let $\psi$ be a biholomorphic (conformal) map from $B(0,1) \backslash\{0\}$ onto $\Omega_{l} \backslash\{q\}$, where $\Omega_{l}$ is the rectangle with vertices

$$
\left(-l+\frac{5}{2} \pi i, \frac{5}{2} \pi i,-l-\frac{1}{2} \pi i,-\frac{1}{2} \pi i\right), \quad l>0, q \in \mathbb{R} \cap \Omega_{l} .
$$

The map $e^{\psi}$ sends $B(0,1) \backslash\{0\}$ onto the annulus

$$
A_{l}=\left\{z: 1 / e^{l}<|z|<1\right\} .
$$

It is a 2 -valent locally biholomorphic map. 
Let $B_{c}, 0<c<1$, denote the domain

$$
B(0,1) \backslash\left\langle 0, c^{-1}\left(1-\sqrt{1-c^{2}}\right)\right\rangle .
$$

We can find $l$ and $c$ such that the domains $A_{l}$ and $B_{c}$ are biholomorphic (conformally equivalent).

Let

$$
\Phi_{c}(z)=z \cdot \frac{z-c}{1-c z} .
$$

It maps $B(0,1)$ onto $B(0,1)$ because it is a Blaschke product. The derivative $\Phi_{c}^{\prime}$ vanishes only at

$$
z=\left(1-\sqrt{1-c^{2}}\right) c^{-1} .
$$

We also have $\Phi_{c}(1)=\Phi_{c}(-1)=1$. Hence $\Phi_{c}$ is a 2 -valent locally biholomorphic map from $B_{c}$ onto the unit disc.

Let $D$ be a domain in $\mathbb{C}$. We shall say that a closed connected set $K \subset$ $\widehat{\mathbb{C}} \backslash D$ is an isolated component of $\widehat{\mathbb{C}} \backslash D$ if there exists an open set $U$ such that $K \subset U$ and $U \backslash K \subset D$.

As a consequence of Proposition 2 we get

Theorem 4. Let $D \subset \mathbb{C}$ be a domain such that $\widehat{\mathbb{C}} \backslash D$ contains an isolated component $K$ not equal to a single point. Then there exists an $m$ valent locally biholomorphic mapping $f$ from $D$ onto the unit disc such that $m \leq 24$.

Proof. By the Riemann theorem there exists a biholomorphic map $h$ from $\widehat{\mathbb{C}} \backslash K$ onto $B(0,1)$. Since $K$ is a component of $\widehat{\mathbb{C}} \backslash K$, the set $h((\widehat{\mathbb{C}} \backslash D) \backslash K)$ is a compact subset of $B(0,1)$. Hence $h(D)$ satisfies the assumptions of Proposition 2.

COROllary 3. For every finitely connected domain $D$ not equal to

$$
\mathbb{C} \backslash\left\{a_{1}, \ldots, a_{M}\right\}, \quad M \in \mathbb{N},
$$

there exists an m-valent locally biholomorphic map $f: D \stackrel{\text { onto }}{\longrightarrow} B(0,1)$ such that $m \leq 24$.

COROllary 4. Theorem 4 is valid for every "Swiss cheese" domain.

We can also prove the following

TheOrem 5. Let $D \subset \mathbb{C}$ be a domain such that $\widehat{\mathbb{C}} \backslash D$ contains a continuum $K$ not equal to a single point, for which the boundary of $\widehat{\mathbb{C}} \backslash K$ is locally connected, and $\widehat{\mathbb{C}} \backslash K$ is connected. Put $K_{1}=\partial(\widehat{\mathbb{C}} \backslash K)$ and assume that $K_{1} \cap \overline{(\widehat{\mathbb{C}} \backslash D) \backslash K} \neq K_{1}$. Then there exists an $m$-valent locally biholomorphic mapping $f$ from $D$ onto the unit disc such that $m \leq 24$. 
Proof. Let $g$ be a biholomorphic map from $B(0,1)$ onto $\widehat{\mathbb{C}} \backslash K$. By the Carathéodory theorem, $g$ extends to a continuous map $\widetilde{g}$ from $\overline{B(0,1)}$ onto $\overline{\widehat{\mathbb{C}} \backslash K}=(\widehat{\mathbb{C}} \backslash K) \cup K_{1}$. By assumption there exists $a \in K_{1}$ and an open neighborhood $U$ of $a$ (in $\widehat{\mathbb{C}}$ ) such that

$$
U \cap[(\widehat{\mathbb{C}} \backslash D) \backslash K]=\emptyset
$$

Hence $\widetilde{g}^{-1}(U)$ is an open set in $\overline{B(0,1)}$ containing boundary points of $B(0,1)$. We have $g^{-1}(U) \subset g^{-1}(D)$. Thus the assumptions of Proposition 2 are satisfied for the domain $g^{-1}(D)$.

Theorems 4 and 5 are very interesting in conjuction with the deep result due to Fornæss and Stout [1]: For every $n$-dimensional paracompact complex manifold $X$ there exists an $m$-valent locally biholomorphic mapping from an $n$-dimensional polydisc onto $X$ such that $m \leq 4^{n}(2 n+1)+2$. This implies the following

THEOREM 6. If a domain $D$ satisfies the assumptions of either Theorem 4 or Theorem 5 then for each connected Riemann surface $X$ (compact or open) there exist $m \in \mathbb{N}$ and an m-valent locally biholomorphic mapping $f$ from $D$ onto $X$ for which $m \leq 12 \cdot 2 \cdot 14=336$.

The following problem seems to be difficult.

Problem 1. Are the conclusions of Theorems 4, 5 and 6 valid for every bounded domain $D$ ?

2. The case of $n>1$. In this part we state some theorems concerning Cartesian products of one-dimensional domains or manifolds.

Our Theorem 2 yields immediately

TheOREM 7. Let $X$ be an $n$-dimensional complex manifold biholomorphic to a Cartesian product of $n$ open Riemann surfaces. Then there exists a locally biholomorphic mapping from $X$ onto $\mathbb{C}^{n}$. Hence $X$ can be represented as a Riemann domain over the whole $\mathbb{C}^{n}$.

A natural question arises: Which Riemann domains $(X, p)$ admit locally biholomorphic mappings from $X$ onto $\mathbb{C}^{n}$ ?

It is easy to see that if $f: X \rightarrow \mathbb{C}^{n}$ is a locally biholomorphic map onto $\mathbb{C}^{n}$ then also its extension $\widetilde{f}: \widetilde{X} \rightarrow \mathbb{C}^{n}$ to the envelope of holomorphy $(\widetilde{X}, \widetilde{p})$ of $(X, p)$ is a locally biholomorphic mapping onto $\mathbb{C}^{n}$.

Hence a correct statement of our problem is the following:

Problem 2. For which Riemann-Stein domains $(X, p)$ over $\mathbb{C}^{n}$ does there exist a locally biholomorphic mapping $f: X \rightarrow \mathbb{C}^{n}$ ?

We conjecture that the answer may be positive for every such $(X, p)$. Our conjecture is motivated by: 
Proposition 3. Let $(X, p)$ be a Riemann-Stein domain over $\mathbb{C}^{n}$. Then for each compact subset $K \subset X$ there exists an almost proper mapping $f: X \rightarrow \mathbb{C}^{n}$ such that $f$ is locally biholomorphic on an open neighborhood of $K$.

Note that an almost proper mapping must be surjective.

Proof. This follows from Theorem VIIC 2 of [5], which says that the almost proper mappings from $X$ onto $\mathbb{C}^{n}$ are dense in $(H(X))^{n}$ in the compact-open topology because $X$ is Stein. Since $X$ is a Riemann domain there exists a locally biholomorphic mapping from $X$ into $\mathbb{C}^{n}$.

There exists a sequence of almost proper mappings $f_{k}$ which tends to $p$ almost uniformly (uniformly on compact sets). Hence each point $x \in X$ has a neighborhood $U_{x}$ such that the Jacobian of $f_{k} \circ p^{-1}$ tends to the Jacobian of $p \circ p^{-1}$ (which is equal to one) uniformly on $p\left(U_{x}\right)$. Thus for every compact $K \subset X$ there exists $k_{0}$ and an open neighborhood $U$ of $K$ such that rank $f_{k}=n$ on $U$ for every $k>k_{0}$.

It should be mentioned here that Fornæss and Stout proved in [2] that the unit ball in $\mathbb{C}^{n}$ can be mapped by an $m(n)$-valent locally biholomorphic mapping onto each $n$-dimensional connected, paracompact complex manifold.

In particular there exists an $m(n)$-valent locally biholomorphic mapping from the unit ball onto $\mathbb{C}^{n}$.

Our Theorems 4, 5 and 6 imply

Theorem 8. Let $X=D_{1} \times \ldots \times D_{n}$ where $D_{i}, i=1, \ldots, n$, satisfy the assumptions of either Theorem 4 or Theorem 5 . Then for each connected, paracompact $n$-dimensional complex manifold $Y$ there exists an m-valent and locally biholomorphic mapping $f$ from $X$ onto $Y$ such that $m \leq$ $(24)^{n}\left[(2 n+1) 4^{n}+2\right]$.

Proof. Theorems 4 and 5 imply that there exists such an $f$ if $Y$ is the unit polydisc in $\mathbb{C}^{n}$. Now the result of Fornæss and Stout [1] implies the assertion.

\section{References}

[1] J. E. Fornæss and E. L. Stout, Spreading polydiscs on complex manifolds, Amer. J. Math. 99 (1977), 933-960.

[2] - - - Regular holomorphic images of balls, Ann. Inst. Fourier (Grenoble) 32 (1982), $23-36$.

[3] G. M. Goluzin, Geometric Theory of Functions of a Complex Variable, Amer. Math. Soc., 1969.

[4] R. C. Gunning and R. Narasimhan, Immersion of open Riemann surfaces, Math. Ann. 174 (1967), 103-108. 
[5] R. C. Gunning and H. Rossi, Analytic Functions of Several Complex Variables, Prentice-Hall, 1965.

[6] M. Jarnicki and P. Pflug, Extension of Holomorphic Functions, de Gruyter, Berlin, 2000.

[7] G. Julia, Leçons sur les fonctions uniformes à point singulier, Gauthier-Villars, Paris, 1924 .

Institute of Mathematics

Warsaw University

Banacha 2

02-097 Warszawa, Poland

E-mail: elig@mimuw.edu.pl

Reçu par la Rédaction le 28.10.2002

Révisé le 21.3.2003 